\title{
A Combined Fractal and Wavelet Angiography Image Compression Approach
}

\author{
Al-Fahoum A* and Harb B \\ Biomedical Systems and Informatics, Engineering Dept., Hijjawi Faculty for Eng. Tech., Yarmouk University, Irbid, \\ 21163, Jordan
}

\begin{abstract}
In this paper, a combined Fractal and Wavelet (CFW) compression algorithm targeting x-ray angiogram images is proposed. Initially, the image is decomposed using wavelet transform. The smoothness of the low frequency part of the image appears as an approximation image with higher self similarities, therefore, it is coded using a fractal coding technique. However, the rest of the image is coded using an adaptive wavelet thresholding technique. This model is implemented and its performance is compared with best performances of the available published algorithms. A data set containing $1000 \mathrm{x}$-ray angiograms is used to study the performance of the algorithm. A minimum compression ratio of 30 with a peak signal to noise ratio (PSNR) of $36 \mathrm{~dB}$ and percent diameter stenosis deviation of $(<0.2 \%)$ was achieved. Results demonstrate the effectiveness of the proposed technique in obtaining a diagnostic quality of reconstructed images at very low bit rates.
\end{abstract}

Keywords: X-ray images, Image compression, Fractal Analysis, Wavelet, Quality Measures.

\section{INTRODUCTION}

Advances in medical image generation made the demand to access, transfer, and interpret medical images in a faster, convenient, and accurate form a necessity. With the inevitable appearance of new medical commercial modalities such as x-ray angiography, CT imaging, MRI, and digital video; compression methods other than those currently utilized within DICOM standard will be necessary to meet requirements of remote medical facilities and to expedite data transmission among different medical centers. Presently, $\mathrm{x}$-ray angiography is the mostly used non-invasive diagnostic and therapeutic technique for vascular diseases. Both diagnosis and therapy of vascular diseases require further processing and proper consultation with other medical centers. Therefore, an image compression that provides diagnostic quality at high compression ratio is vital. In recent decades, cardiologists and vascular surgeons have repaired the blood vessels and arteries of the heart using $\mathrm{x}$ ray angiography procedure. Recently, $x$-ray angiography is utilized for therapy. However, in third world countries conventional x-ray angiography still has the major role in the detection, diagnosis and treatment of heart disease, heart attack, acute stroke and vascular disease.

Due to the large size of population who are diagnosed on daily basis to have different types of vessel problems, the need for reliable remote medical centers is imperative, and also because Coronary artery disease is the major cause of premature death in the United Kingdom [1], reducing the amount of data necessary to be sent based on an on-line

*Address correspondence to this author at the Biomedical Systems and Informatics Engineering Dept., Hijjawi Faculty for Eng. Tech., Yarmouk University, Irbid, 21163, Jordan; Tel: +962 2 7211111; Fax: +96227274725; Emails: afahoum@yu.edu.jo; amjed.alfahoum.rcap@gmail.com scheme is necessary and requires an efficient medical image compression algorithm that is able to reduce the bit rate of transmission and to maintain the diagnostic quality of the images. On the other hand, dealing with the angiogam as it is requires higher storage memory or higher bandwidth for transmission. For example, a single patient digital angiogram video typically requires $7.5 \mathrm{Mbytes} / \mathrm{s}$ for $512 * 512 * 8$-bit resolutions at 30 frames/s, resulting in $0.25 \mathrm{Mbytes} /$ frame [2].

There are several techniques that have been used for medical image compression, but those that maintain the diagnostic ability for an image with an increase in compression ratio are required. Bearing in mind, the aim is to achieve a better compression ratio with minimal distortion requires the existence of reliable metrics to measure the diagnostic distortion rather than quantitative distortion. It also requires knowledge of the standards of image quality and how the compression technique is handled in digital medical imaging device. The most prime measures to be taken into considerations and must be correctly satisfied are: Peak Signal-to-Noise Ratio (PSNR), Signal-to-Noise Ratio (SNR), Peak Error (PE), Percent Root Mean Square Deviation (PRMSD), Normalized Sum Of Scores (NSOS), and Cross Correlation (CC).

Among compression techniques, recently two techniques are found to be the best in maintaining image quality and increasing compression ratio, these are Wavelet and Fractal techniques.

Wavelet analysis has generated much interest in both theoretical and applied mathematics over the past decade. It is a time-scale representation that has been used successfully in a wide range of applications. Wavelet transformation consolidates image energy in few coefficients with excellent localization characteristics in the spatial-frequency domain [3]. To compress the image, these coefficients will go 
through a thresholding process depending on the criterion chosen to ignore the less significant coefficients. For reconstruction, an inverse Wavelet transform to the thresholded coefficients is applied. The result will lead to a compression ratio lower than 1.4 for an original angiogram image with different biorthogonal filters [4]. The problem with this transform is that it needs a very high threshold for the approximation coefficients; hence no compression is achieved for these coefficients, this is considered as a constrain point for this method.

Fractal Compression was first promoted by M. Barnsley [5]. Fractal encoding depends on the assumption that natural and most artificial objects contain similar redundant repeating patterns called fractals [6]. The basic idea of fractal transform was built on the self-similarity of the objects that allows saving a small portion of the object instead of the whole object. The problem in fractal compression is its time consumption, where it is very slow in analyzing the data for large images.

A combined 'Fractal and Wavelet' (CFW) compression algorithm is proposed in this paper. It maximizes the advantages of Fractal and Wavelet methods and gets rid of their disadvantages. However, combining wavelet and fractal is not new but it depends on utilized criteria for hybriding. One of these techniques is based on compressing high frequency wavelet coefficients by using a modified fractalcoding algorithm and coding the low frequency wavelet coefficients by a lossless method called BTPC [7]. Another criterion is introduced in the Hybrid Fractal Zerotree Wavelet Image Coding which couples a zero tree-based encoder, such as the embedded zerotree wavelet (EZW) coder or set partitioning in hierarchical trees SPIHT and a fractal image coder [8]. The idea is based on applying a locally optimal distortion-rate calculation for trading in similar structures in EZW and tree-based fractal encoders. The coder that performs efficiently is adaptively selected. A fractal coder usually performs better with edges and texture areas of an image [9]. The best achieved PSNR ranged between $26.49 \mathrm{~dB}$ and $39.28 \mathrm{~dB}[8]$.

The rest of the paper is divided into five main sections: compression techniques, proposed approach, quality of decompressed images, achieved results, discussions and conclusions. Section 2 covers the recent developments in fractal and wavelet based image compression and coding techniques. Section 3 lays out the foundation for the proposed combined fractal wavelet compression approach and it is implementation. Section 4 introduces quality performance measures that will be used to validate the proposed approach. It discusses both subjective and objective quality measures. Implementation and results are covered in section 5. Manifestation and efficacy of the proposed technique are highlighted. Moreover, comparisons among medical image compression techniques are also studied. Section 6 explains the obtained results and concludes main achievements of this codec followed by references.

\section{IMAGE COMPRESSION TECHNIQUES}

The era of advanced communication systems, satellite communications, and mobile telecommunication paced the birth of new research in image compression literature.
Wavelet and fractal image compression techniques gained further momentum in its development for their ability in producing high compression rates with perceptually relevant quality. Advances in both compression areas are considered as the base for this current research. Therefore, background analysis of recent emerged compression techniques in wavelet and fractal domains will provide an insight to the proposed technique and strengthen its achievements.

\subsection{Image Compression in Wavelet Domain}

Wavelet is the base of multiresolution image decomposition which allows efficient subband coding [10]. It reveals strong space-frequency localization where relevant features within each subband and similar features across subbands are clustered [11]. Wavelet subbands are statistically dependent making it very suitable for efficient coding [12]. The pyramidal, or dyadic wavelet decomposition, in particular, illustrated excellent energy compaction. EZW encoder is the first to utilize this property. It uses both bit-plane coding and the zerotree configuration [13]. Said and Pearlman partitioned the embedded tree and proposed the set partitioning in hierarchical trees (SPIHT) encoder [14]. EZW has excellent rate-distortion (R-D) performance and low computational complexity EZW coders are progressive coders in which data can be transmitted and encoded at any target bit rate. However, exploiting the wavelet tree in adaptive manner could improve the compression results and provide low rate at a higher computational costs. This Adaptive concept is employed non-progressively in Xiong et al. [15]. Although their algorithm provides better compression results compared with non-adaptive EZW coders its computational complexity is extremely high. It is worth mentioning that both types of coders are still focusing on the inter-subband correlation. Another type of coders that base their compression on a structure-like mechanism is recently evolved. The layered zero coding (LZC) algorithm proposed by Taubman and Zakhor [16] for still and video images represents a good example of structure-like compression approach that efficiently incorporates an adaptive arithmetic coding [17]. Other algorithms that exploit the wavelet domain do exist among these algorithms are those that concentrate on the morphological representation [18, 19], classification of image subbabands [20], and estimating the statistical properties of coefficients in the subbands [21,22]. The latter are called backward adaptive techniques and demonstrated superior rate-distortion performance in comparison with forward adaptive ones. However, they still suffer from higher computational complexity at the decoder side.

\subsection{Fractal Image Compression and Wavelet Domain}

Fractal image compression is based on exploiting the self similarity between different image patterns. Redundant information within the spatial image is effectively reduced using this technique $[23,24]$. Recent results showed that applying fractal coding in wavelet domain using self similarities within and across wavelet trees gives better compression results compared to classic compression schemes as well as simple fractal coding schemes. This is due to its capabilities to eliminate blocking effect and to provide more efficient coding for the quantized coefficients. 


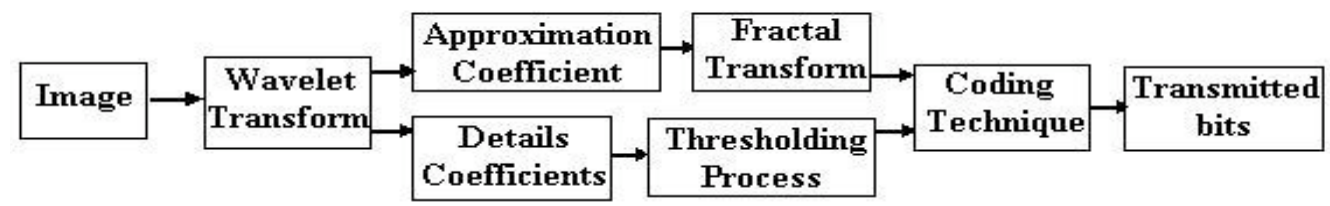

a) Compression Encoder

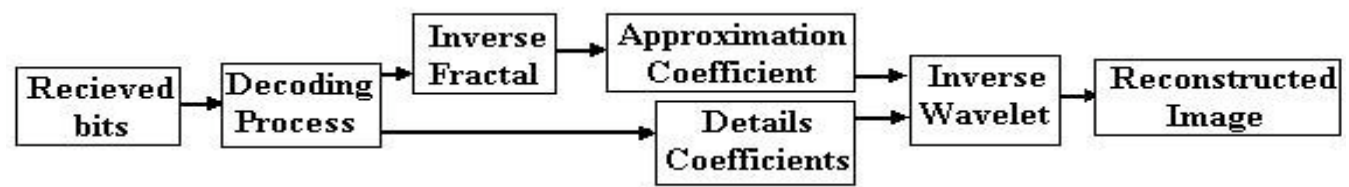

b) Compression Decoder

Fig. (1). Outline of the Proposed CFW image compression approach.

On the other hand, the long time required to generate simple coding and codebook is considered inefficient when compared to wavelet methods. To reduce the encoding time, selected patterns are classified using different classification techniques in an attempt to rule out detected redundant blocks from the codebook.

Pentland and Horowitz [25] are the first who introduced the study of the self-similarity in a subband decomposition which is later compressed using fractal coding methods by Rinaldo and Calvagno [26]. A generalization of the previous method in wavelet domain is proposed by Davis [27]. In his work, Davis demonstrated that self similar zerotree-like structures and straight edges are efficiently encoded. Based on Davis work, Li and Kuo proposed a hybrid waveletfractal image coder which has better quality and speed [28]. Zoukouski et el., depending on radiologist judgment, used Lossless compression in clinically relevant regions and lossy compression for other regions [29]. Recently several research articles combined wavelet compression with linear predictive coding to predict the behavior of compressed wavelet coefficients [30-33]. However, linearity in Wavelet domain is purely nonlinear in spatial domain. Singh et. el. proposed a hybrid technique involving both DWT and DCT to compress medical images [34]. This two-step algorithm is expensive and suffers coding problems. In summery, available wavelet based fractal coding methods depend on predicting blocks from the coarse levels to upper detail ones incorporating achievements of either EZW, SPIHT, or LZC coders. The same strategy for compression is still studied, applied, and narrowly verified. In this paper, we propose a combined fractal morphological wavelet coder (CFW) coder. The new coder aims at further exploiting the strengths of the fractal image compression and best utilization of the energy compaction property of the wavelet domain.

Fig. (1) illustrates the block diagram of the proposed compression scheme. Initially, the image is decomposed into its spatial-frequency domain using wavelet transform. Then the low frequency part of the decomposed image is coded using a variable-size fractal coding technique while the rest of the image is coded using an embedded zero wavelet (EZW) technique. Therefore, the proposed algorithm solves the inherited problems of both fractal and wavelet coders. Self similarity is emphasized at low frequencies and EZW coder works efficiently at higher frequencies.

\section{COMBINED FRACTAL WAVELET COMPRE- SSION APPROACH}

The proposed compression approach, as shown in Fig. (1), depends on maintaining the properties of wavelet transform coders in minimizing the bit quota for nonsignificant coefficients and maintaining the most significant parts of the wavelet subbands at lower costs. Moreover, it is expected to utilize the efficiency of fractal image compression in detecting similarities and their efficient encoding. In the following subsections the wavelet decomposition procedure, fractal image compression, and proposed encoding scheme are further explained and clarified.

\subsection{Compression of the High-Resolution Subbands in Wavelet Domain}

Natural images usually have smooth color variations, with the fine details appear as sharp edges in between the smooth variations. Technically, smooth objects in an image represent low frequency contents while fine details represent high frequency variations. Decomposing the image into its smooth variations and details can be obtained simply by using a Discrete Wavelet Transform (DWT) [10]. The decomposition process is divided into several levels. Each level will produce a two-dimensional array of coefficients contains four bands of data. These bands are labeled as LL (low-low), HL (high-low), LH (low-high) and HH (highhigh) respectively. The next level will decompose the LL band again in the same manner, thereby producing even more sub bands. This process can be repeated up to any level, thereby resulting in a pyramidal decomposition. Fig. (2) shows a five-levels wavelet decomposition of an angiogram image.

The LL band at the upper level of decomposition is classified as the highest energy band with smooth visitations, and the other 'detail' bands are classified as lower energy bands with sharp variations. As it is obvious from Fig. (2), the degree of energy compaction is decreasing from the top of the pyramid to the bands at the bottom [10]. Moreover, further decomposition will not reveal further information regarding the image. After decomposing the image into sublevels, a stream of coefficients will appear. A few of these coefficients contain the most energy of the image while others appear as less significant details. Hence, the aim is to extract the significant coefficients, which contain most of the 


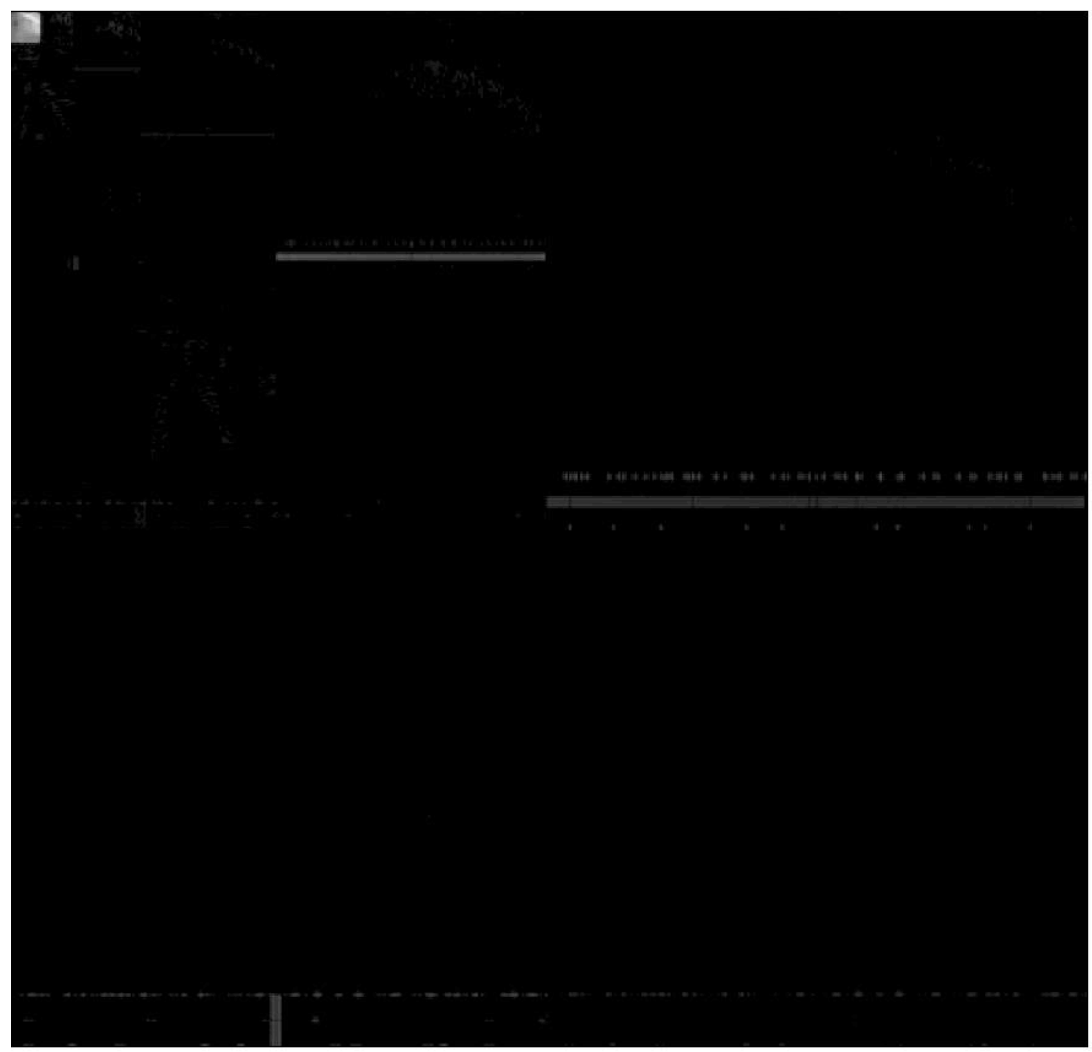

Fig. (2). Five Levels Pyramidal Decomposition of an Angiogram.

energy, and ignore the others. Regardless of the adopted compression scheme in the wavelet domain, if used as is, the low-resolution subband which contains most of the energy of the image and characterized by its smooth variation will consume most of the bit quota. Therefore, to effectively utilize the properties of the fractal image compression this part is coded separately using fractal image coding. Other parts of the image that contain more coefficients but at low amplitude levels will be coded efficiently using the wavelet domain only. To achieve this, after decomposing the image signal into sublevels an appropriate threshold (thr) is needed to control the compression rate and the quality of the detailed sub images. Thresholding is a process that compares some given values to a reference value (thr). A hard thresholding concept is used in compressing the details of the image which is described as setting the values that are less than the reference value to zero, and keeping the other values, which are greater than this value, to their original value. Hard thresholding process is illustrated as follows, assume that $x(n)$ is a detailed wavelet coefficient and $x^{\prime}(n)$ is the coefficient after thresholding:

$\left\{\begin{array}{l}\text { If }|x(n)|>t h r \\ x^{\prime}(n)=x(n) \\ \text { Else } \\ x^{\prime}(n)=0\end{array}\right.$

After defining the thresholding process, the criterion for finding the threshold value (thr) is the next step. Different techniques are used to calculate the threshold value. One of these techniques is based on the energy requirements. Therefore the way in choosing the threshold value depends

on the total energy of the detailed sub images. In order to calculate a threshold based on a certain portion of the energy, the following steps are performed:

- Calculate the total energy of each subband, $E_{T i}$, using the wavelet coefficients $\mathrm{x}[\mathrm{n}]$, as follows:

$\mathrm{E}_{\mathrm{Ti}}=\sum_{n=1}^{L i} x^{2}(n)$

- Calculate the desired energy in the thresholded coefficients, $\mathrm{E}_{\mathrm{p} \text {, }}$, i.e.:

$\mathrm{E}_{\mathrm{Pi}}=\sum_{n=1}^{P i} x^{2}(n)$

- To determine the value pi and the threshold, the following steps are conducted:

a. Sort the wavelet coefficients in descending order based on its energy, $\mathrm{x}_{\text {sort }}[\mathrm{n}]$.

b. Apply the following procedure:

Set the portion of the retained energy $=$ Percentage of $E_{T}$

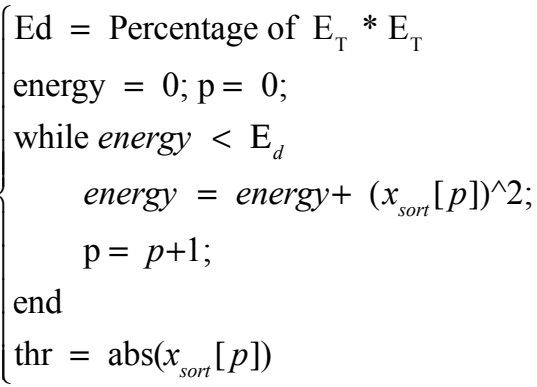




\begin{tabular}{|r|r|r|r|r|r|r|r|r|}
\hline $\mathrm{R} 11$ & $\mathrm{R} 12$ & $\mathrm{R} 13$ & $\mathrm{R} 14$ & $\ldots$ & $\ldots$ & $\ldots$ & $\ldots$ & $\mathrm{R} 1 \mathrm{n}$ \\
\hline $\mathrm{R} 21$ & $\mathrm{R} 22$ & $\mathrm{R} 23$ & $\mathrm{R} 24$ & $\ldots$ & $\ldots$ & $\ldots$ & $\ldots$ & $\mathrm{R} 2 \mathrm{n}$ \\
\hline$\ldots$ & $\ldots$ & $\ldots$ & $\ldots$ & $\ldots$ & $\ldots$ & $\ldots$ & $\ldots$ & $\ldots$ \\
\hline$\ldots$ & $\ldots$ & $\ldots$ & $\ldots$ & $\ldots$ & $\ldots$ & $\ldots$ & $\ldots$ & $\ldots$ \\
\hline$\ldots$ & $\ldots$ & $\ldots$ & $\ldots$ & $\ldots$ & $\ldots$ & $\ldots$ & $\ldots$ & $\ldots$ \\
\hline$\ldots$ & $\ldots$ & $\ldots$ & $\ldots$ & $\ldots$ & $\ldots$ & $\ldots$ & $\ldots$ & $\ldots$ \\
\hline$\ldots$ & $\ldots$ & $\ldots$ & $\ldots$ & $\ldots$ & $\ldots$ & $\ldots$ & $\ldots$ & $\cdots$ \\
\hline $\mathrm{Rm} 1$ & $\mathrm{Rm} 2$ & $\mathrm{Rm} 3$ & $\mathrm{Rm} 4$ & $\ldots$ & $\ldots$ & $\ldots$ & $\ldots$ & $\mathrm{Rmn}$ \\
\hline
\end{tabular}

Fig. (3). Segmenting an image into a set of range blocks.

If the overall distortion is within the accepted ratedistortion criterion, significant coefficients will be separately encoded using adaptive differential run length and progressively transmitted. Otherwise, other percentage is used and the same procedure is repeated until the criterion is satisfied [35].

\subsection{Compression of Low-Resolution Subband Using Fractal Image Compression}

Efficiency of fractal transform coder is gained due to the availability of self-similarity of the objects allowing the coder to reduce the needed space for the whole object and only code a small portion of it. Self-similarity is not fully maintained in natural images, however, some portions of the image are self similar. Lena image, for example, does not contain the type of self-similarity which is found in the Sierpinski triangle [5]. However, self-similar portions of the image are clear in it. These detected parts are not exactly the same due to the affine transformation process applied to them. This means that the results of the encoding process will not be an identical copy of the original image. However exploiting self similarities within the whole image would effectively reduce its desired bit quota. On the other hand, if the image is decomposed using wavelet transform and lower resolution portion of it is utilized for fractal encoding, the encoder will be more efficient due to the maximized similarities within smooth objects and to their energy compaction dominance. Moreover, to resolve the non identical coding of similar objects due to transformations, a segmentation process is proposed. Segmentation will further enhance the performance of the fractal coder. Fractal compression for low resolution wavelet image is conducted as follows:

\subsubsection{Encoding Scheme}

\subsubsection{Segmentation}

An image is regularly segmented into blocks. Fig. (3) shows the segmentation of the image into range blocks. Each block is a two-dimensional array of BxB pixel. These blocks are called range blocks. Each range block is arranged in a 1-
D sequence of a row followed by another row order. The whole $2 \mathrm{D}$ array of range blocks is then represented by $1 \mathrm{D}$ sequence, i.e. $\mathfrak{R}=\left\{R_{i}\right\}[7]$.

\subsubsection{Creating Domain Block Pool}

The pool of the set of domain blocks contains $2 \mathrm{Bx} 2 \mathrm{~B}$ squares covering the whole of the original image. This pool is generated by sliding a $2 \mathrm{Bx} 2 \mathrm{~B}$ window within the original image and skipping $\Delta$ pixels from left to right, top to bottom. Also each domain block is transformed in 8 different ways. So, this will produce a huge pool of domains.

\subsubsection{Affine Map}

For each range block, a domain block and map need to be specified, so that range block and domain block become the best pair. To minimize the delay due to calculations, the domain block is down sampled to be equal to the range block size of $\mathrm{BxB}$ pixels. For each best pair of range and domain mapping coefficients are saved. These coefficients include the location of the domain, the transformation type of the domain, the contrast of the domain, and the offset of the range.

\subsubsection{Extracting the Non-Similar Part}

In the case where a block is uniquely identified, each range block that does not pair with a domain block is marked as a non-similar part. To avoid losing such data, all of these ranges are losslessly coded and sent as is. This would introduce an overhead problem if the number of non similar blocks covers the whole image. Such situation is almost impossible.

\subsubsection{The Decoding Scheme}

The contractive mapping theorem is utilized to decode the image. The decoding process starts by any image, for example rectangle. The decoder maps the mapping coefficients to the initial seed image iteratively. This process converges to fixed point of the mapping coefficients, once the compressor has found good mapping coefficients for the image. To perform an iteration of the mapping coefficients, 
the decoder takes the list of all affine maps and applies each one in turn. This converts a set of domains into a set of ranges. Since no overlapping is allowed between range blocks, the whole image will be covered. Therefore, a new complete image is produced as a result. The decoder will repeat the whole process until convergence is achieved. That is, until there are a little difference between the output of the current iteration and its input image. Convergence is usually very fast and is obtained by 3 to 6 iterations.

\section{QUALITY OF THE RECONSTRUCTED IMAGES}

The quality of the reconstructed image and the compression ratio determine the performance of the compression scheme. Due to the reduction of the CFW coefficients the reconstructed signal will suffer a reduction in its quality. Therefore there must be some quality measures that evaluate the quality of the reconstructed image. Two types of evaluation mechanisms are found in the literature and utilized to validate the efficacy of the proposed algorithm [36].

\subsection{Subjective Evaluation Technique}

In this part, an observer is asked to evaluate the compression algorithm by following some rules. However, variations among different observers recommend applying objective measures that will rate the quality based on mathematical formula.

\subsection{Objective Quality Measures}

Several measures can be incorporated to evaluate the performance of the proposed compression algorithm. In this paper the following measures are used:

\subsubsection{Calculating the Peak Signal to Noise Ratio (PSNR):}

Calculating the PSNR gives a clear mathematical evaluation of the amount of destruction affected to the original signal. The calculation of the PSNR is as follows:

$$
P S N R=10^{\prime} \log _{10}\left(\frac{255^{2}}{\text { variance }\left(x-x_{r}\right)}\right)
$$

where $x$ is the original signal, and $x_{r}$ is the reconstructed signal. The 255 represents the highest level of an 8 bit image. For other image format, it will be $\left(2^{\mathrm{Nb}}-1\right)$, where $\mathrm{Nb}$ is the number of pits/pixel of the image.

\subsubsection{Calculating the Signal to Noise Ratio (SNR)}

The SNR is an important measure for any system, it simply indicates the ratio of the input signal to the noise, which is always must be high enough. The calculation of the SNR is as follows:

$\mathrm{SNR}=-10 \times \log _{10}\left(\frac{\operatorname{var} \operatorname{iance}\left(x-x_{r}\right)}{\operatorname{var} \operatorname{iance}(x)}\right)$

\subsubsection{Calculating the Peak Error (PE)}

Peak error provides the maximum available error. A reduced value indicates that the distortion is reduced. Calculation of the PE is defined as follows:

$\mathrm{PE}=\max \left(x-x_{r}\right)$

\subsubsection{Calculating the Partial Root Mean Square Difference (PRMSD)}

The calculation of the PRMSD is as follows:

$\operatorname{PRMSD}=\sqrt{\frac{\operatorname{var} \operatorname{iance}\left(x-x_{r}\right)}{\operatorname{var} \operatorname{iance}(x)}}$

\subsubsection{Calculating the Normalized Sum Of Scores (NSOS)}

The calculation of the NSOS is done as follows:

$\operatorname{NSOS}=\frac{\sum\left(\mathrm{x}-\mathrm{x}_{\mathrm{r}}\right)^{2}}{\sum x^{2}}$

\subsubsection{Calculating the Cross Correlation (CC)}

It measures the amount of similarity between original and reconstructed signal. It is limited in absolute form between zero and one. When the value is very close to one it means that the two images are identical as it goes toward zero it means that images are dissimilar. The calculation of the $\mathrm{CC}$ is done as follows:

$$
\mathrm{CC}=\frac{\sum\left(\mathrm{x} \times \mathrm{x}_{\mathrm{r}}\right)}{\sqrt{\sum \mathrm{x}^{2}} \sqrt{\sum x_{r}^{2}}}
$$

\section{IMPLEMENTATION AND RESULTS}

In this section, the proposed simulation model used in computer implantation is described. The results obtained will also be presented.

\subsection{Implementation}

The CFW algorithm is implemented on Matlab 7.0 where a graphical user interface GUI application is created. Fig. (4) shows the simulation model. The software initially calls the angiographic image to be compressed. Three compression approaches are available: wavelet, fractal, and combined fractal wavelet. The package enables the use of both orthogonal and bi-orthogonal wavelets. All the parameters in the package is either automatically selected to produce the best R-D or manually selected by the user. The package provides rate and objective quality measures for the reconstructed image and allows the generation of a report of the results.

\subsection{Results}

To demonstrate the capability of the proposed CFW coder in providing proper compression ratio at an acceptable image quality, a randomly selected angiogram is used. The angiogram is compressed using the designed package. The user can either use a global threshold that is obtained using equation 4 or local thresholds per sub-band as clarified in section 3.1. Moreover, the package can decompose the image into any specified number of levels by using either available wavelet filters or specifically provided wavelet filters. For demonstration purposes a 4-level wavelet decomposition is performed using bior4.4 wavelet filters. The result of applying the CFW coder of this example is shown in Fig. (5). It is worth mentioning that the global threshold is the minimum maintained detailed wavelet coefficient. Other 


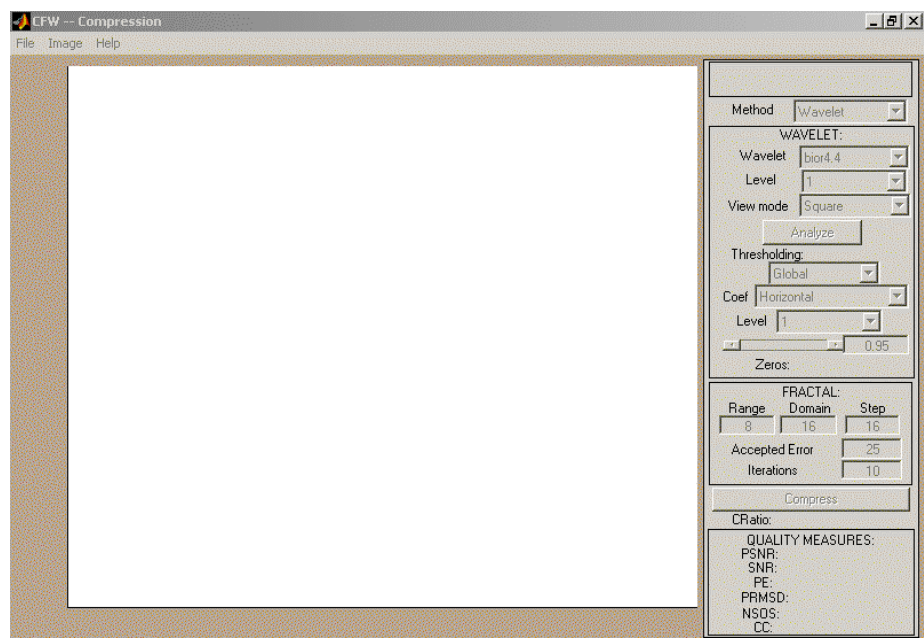

Fig. (4). Graphical user interface of the CFW algorithm.

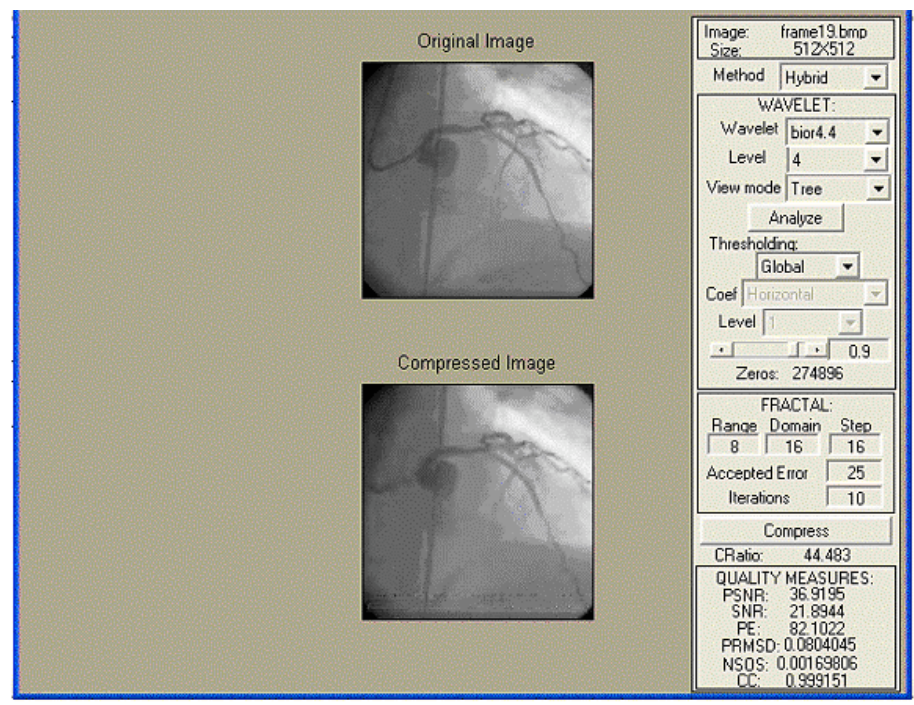

Fig. (5). Demonstration of the CFW on an angiogram image. Both original and compressed images are shown.
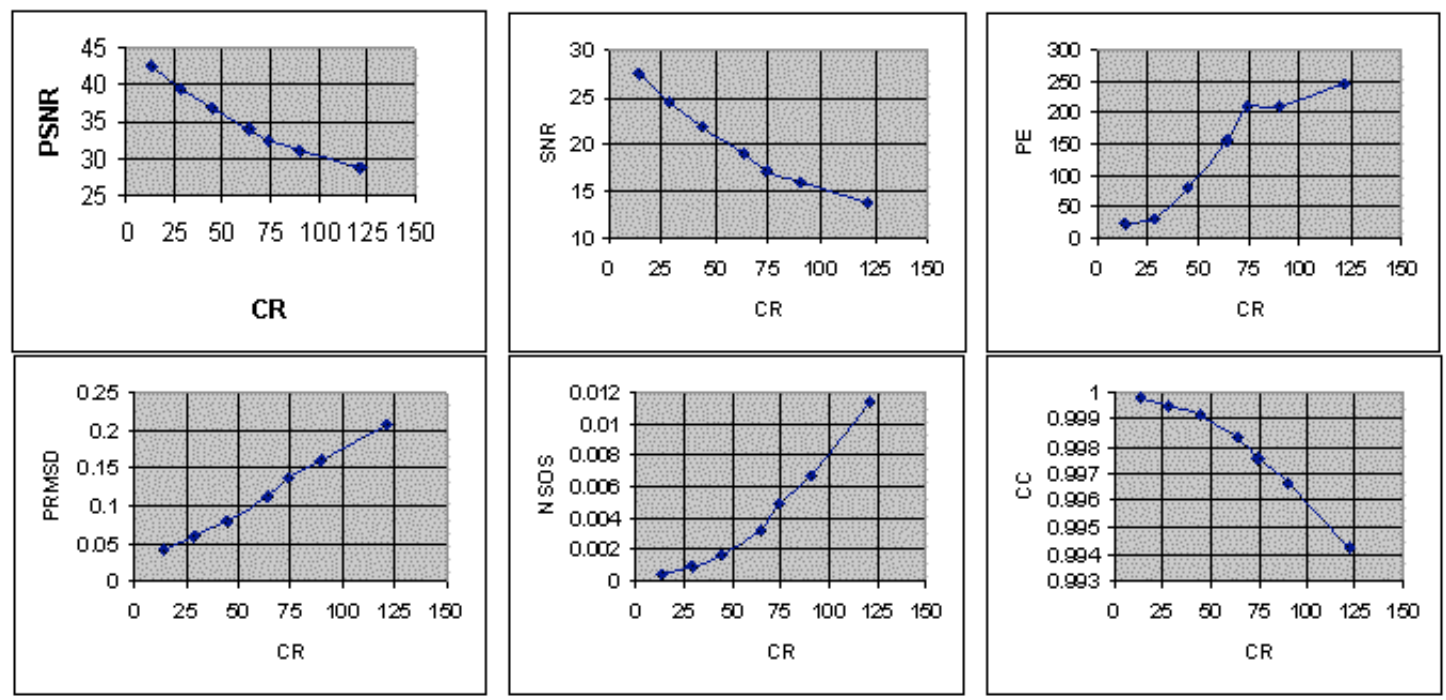

Fig. (6). Performance of CFW coder using different quality measures.

coefficients are considered non-significant. To study the impact of the global threshold on the results obtained using the CFW coder. Fifty images are compressed using CFW coder. The wavelet coder uses the bior 4.4 wavelet filter with 4 levels. The fractal coder uses range $=8$, Domain $=16$, step size $=16$, and an acceptable error $=25$. For each global 
Table 1. Impact of Global Thresholds and Quality Measures of CFW Coder

\begin{tabular}{|c|c|c|c|c|c|c|c|}
\hline Threshold & CR & PSNR & SNR & PE & PRMSD & NSOS & CC \\
\hline \hline 0.4 & 121.5247 & 28.6402 & 13.61514 & 245.0201 & 0.208566 & 0.011426 & 0.994271 \\
\hline 0.6 & 90.07224 & 30.97962 & 15.95456 & 209.7514 & 0.159321 & 0.006667 & 0.996661 \\
\hline 0.7 & 74.42251 & 32.26719 & 17.24213 & 210.0792 & 0.13737 & 0.004957 & 0.997519 \\
\hline 0.8 & 63.96291 & 34.0354 & 19.01034 & 156.3322 & 0.112068 & 0.003299 & 0.99835 \\
\hline 0.9 & 30.3451 & 36.91945 & 21.8944 & 82.10223 & 0.080404 & 0.001698 & 0.999151 \\
\hline 0.95 & 28.49391 & 39.56293 & 24.53788 & 33.91493 & 0.059307 & 0.000924 & 0.999539 \\
\hline 0.98 & 13.68077 & 42.50832 & 27.48327 & 22.90599 & 0.042251 & 0.000469 & 0.999766 \\
\hline
\end{tabular}

Table 2. The PSNR for Different Compression Method at Various Bit Rates

\begin{tabular}{|c|c|c|c|c|c|}
\hline \multirow{2}{*}{ Image Type } & \multirow{2}{*}{$\begin{array}{c}\text { Bit Rate } \\
(\mathbf{p b b})\end{array}$} & SPIHT & JPEG2K & DCT-CSPIHT & Proposed CFW \\
\cline { 2 - 6 } & 0.025 & 33.4 & 36.1 & 39.5 & 40.3 \\
\hline \hline \multirow{3}{*}{ Angiogram } & 0.10 & 40.3 & 44.1 & 45.2 & 45.8 \\
\cline { 2 - 6 } & 0.24 & 45.4 & 47.6 & 48.4 & 58.9 \\
\cline { 2 - 7 } & 0.50 & 49.8 & 50.5 & 52.0 \\
\hline
\end{tabular}

threshold all the objective quality measures of section 4 are calculated. Table 1 summarizes the results for different threshold values. As it can be noticed from the table higher compression ratios are found at acceptable reconstructed image qualities. Fig. (6) illustrates the functional performance of the coder using different quality measures. Performance is acceptable when compared with recently published results. The performance of the proposed algorithm is evaluated using a $\mathrm{PC}$ with $\mathrm{AMD} /$ Athlon 2.0 $\mathrm{GHz}$ processor. To show the capability of the proposed algorithm in comparison with well known compression schemes, the algorithm is compared with SPIHT, JPEG2000, and DCT-CSPIHT respectively [14], [37, 38]. Table 2 shows the PSNR for different compression methods at various Bit rates. As it is clearly obvious from Table $\mathbf{2}$, the proposed algorithm performs better than all of the considered algorithms. Table 2 shows a comparison between the results of the proposed algorithm and those results achieved by other compression algorithms. As it can be depicted from table 2, the proposed algorithm provides better qualities for the same results. CFW outperforms SPIHT, JPEG2000, and DCT-CSPIHT respectively. The result clearly demonstrates that the incorporation of fractal compression provides a substantial performance improvement for the wavelet coder. Another important aspect of Image compression is the speed of encoding and decoding of the compression algorithm. 50 images are used to study this parameter. Table $\mathbf{3}$ shows a comparison of the encoding/decoding speeds for different algorithms [39]. To study the impact of the wavelet filter on the performance of the algorithm, 100 images are used to test the performance of the algorithm at a compression rate of 40 . Four different wavelet filters are used. The results are summarized in Table 4. As shown in Table 4, the Bior4.3 filter has the best PSNR at $32.76 \mathrm{~dB}$. To study the effect of the compression scheme on the quality of x-ray angiograms, a $1000 \mathrm{x}$-ray images are compressed using the compression algorithm such that a minimum PSNR is $36 \mathrm{~dB}$. The minimum compression ratio achieved was 30 . The reconstructed images are then evaluated using Quantitative coronary Analysis (QCA) to determine the effect of compression on stenosis. The maximum deviation between the original and compressed diameter was less than $0.2 \%$ [40].

\section{DISCUSSION AND CONCLUSIONS}

Wavelet-based coders are initially introduced, discussed and implemented. Fractal image compression techniques are also investigated and implemented. A combined fractal wavelet image coding algorithm is then proposed. It couples morphological wavelet and fractal image coding. The new coder represents the cream of the cream of fractal and wavelet coders with a superior rate distortion performance. The coder was purposely designed to compress $\mathrm{x}$-ray angiograms to satisfy feasible medical requirements at lower bit rates. The coder doesn't suffer complex coding structure, on the contrary, it allows simple reconstruction and includes a developed rule to estimate the amount of overhead information and the amount of bit rate savings achieved by applying the fractal coding for the low resolution wavelet sub image. In case such overhead is high or no bit rate saving is achieved only lossless wavelet coder is used. Detailed wavelet coefficients in other regions are encoded directly with successive quantization and context-based variable run length encoders. It should be mentioned that a rate-distortion criterion is used to achieve maximum bit rate saving. The optimization provided the optimal fractal parameter that will produce best rate distortion trade off. CFW outperforms JPEG, typical fractal and wavelet coders. 
Table 3. The Average Encoding/Decoding Time in Seconds

\begin{tabular}{|c|c|c|c|}
\hline \multicolumn{3}{|c|}{ Algorithm Method } \\
\hline SPIHT & JPEG2000 & WCAP40 & Proposed CFW \\
\hline \hline $2.3 / 2.7$ & $1.1 / 1$ & $5.4 / 2.9$ & $1.0 / 0.87$ \\
\hline
\end{tabular}

Table 4. The Average PSNR Values in dB Using Different Wavelet Filters at Compression Ratio of 40:1

\begin{tabular}{|c|c|c|c|c|}
\hline \multirow{2}{*}{ Image Type } & \multicolumn{4}{|c|}{ Wavelet Filter } \\
\cline { 2 - 5 } & Symlets 5 & Bior 4.3 & Harr & Daubechies 3 \\
\hline \hline 100 Angiograms & 32.68 & 32.76 & 31.24 & 32.37 \\
\hline
\end{tabular}

Results clearly demonstrate that the incorporation of fractal compression provides a substantial performance improvement for the wavelet coder.

This new algorithm efficiently reduces the required bit rate to achieve the desired level of perceptual image quality. It also maintains advantages and properties of both types of coders. It allows online transmission, block mapping, and doesn't inherit the shortcomings of other HFW encoders that are computationally intensive in their encoding part. This is due to the down sampling process for the domain block. Furthermore it doesn't suffer from blocking artifacts produced in JPEG like coders. The proposed CFW uses a constant range-domain block size $\mathrm{K}$. Using variable block size may improve the performance on the account of complexity. For practical applications, it is important to reduce the complexity of fractal search. This is achieved by reducing the size of the domain pool. Also it may be achieved by adopting fast fractal search schemes with little additional degradation. Our future work will concentrate on performing a thorough study on the trade of complexity reduction and the degradation of the R-D performance.

\section{CONFLICT OF INTEREST}

The authors confirm that this article content has no conflicts of interest.

\section{ACKNOWLEDGEMENTS}

Declared none.

\section{REFERENCES}

[1] Grech ED, Ramsdale DR. Practical interventional cardiology. London, UK: Martin Dunitz Ltd 1997.

[2] Joonmi Oh, Sandra IW, Theodoros NA, John NT. A multistage perceptual quality assessment for compressed digital angiogram images, IEEE Trans Med Imaging 2001; 20: 1352-61.

[3] Strang G, Nguyen T. Wavelets and filter banks. Cambridge, MA: Wellesley-Cambridge Press 1997.

[4] Joonmi O, Sandra IW. Reversible wavelet compression for digital angiograms. IEMBS 1998; 3: 1442-45.

[5] Literature Number: BPRA065, Texas Instruments Europe, An Introduction to Fractal Image Compression. October 1997.

[6] Fractal Image Compression. Available at: http://inls.ucsd.edu/y/Fractals/, Yuval Fisher's fractal image compression page, http://www.rocq.inria.fr/fractales/, Grouped Fractals.

[7] Andreopoulos I, Karayiannis YA, Stouraitis T. A hybrid Image compression algorithm based on fractal coding and wavelet transform. ISCAS 2000; 3: 37-40.
[8] Taekon K, Robert EVD, David JM. Hybrid fractal zero tree wavelet image coding. Signal Proc-Image. 2002; 17 (4): 347-60.

[9] Wenxiu F, Shigang W, Dahui K. Hybrid fractal object-based zerotree wavelet video coding algorithm. ICSP '04 2004; vol. 2: pp. 1147-50.

[10] Woods JW, O'Neil DD. Subband coding of images. IEEE Trans Acoust Speech 1986; 34: 1278-88.

[11] Antonini M, Barlaud M, Mathieu P, Daubechies I. Image coding using wavelet transform. IEEE Trans Imaging Proc 1992; 1 (2): 205-20.

[12] Buccigrossi RW, Simoncelli EP. Image compression via joint statistical characterization in the wavelet domain. IEEE Trans Image Proc 1999; 8: 1688-701.

[13] Shapiro JM. Embedded image coding using zero trees of wavelet coefficients. IEEE Trans Signal Process 1993, 41: 3445-62.

[14] Said A, Pearlman WA. A new, fast and efficient image codec based on set partitioning in hierarchical trees. IEEE Trans Circ Syst Video Technol 1996; 6 (3): 243-50.

[15] Xiong Z, Ramchandran K, Orchard MT. Space-frequency quantization for wavelet image coding. IEEE Trans Image Proc 1997; 6: 677-93.

[16] Taubman D, Zakhor A. Multirate 3-D subband coding of video. IEEE Trans Image Proc 1994; 3(5): 572-88.

[17] Witten IH, Neal M, Cleary JG. Arithmetic coding for data compression. Commun ACM 1987; 30: 520-40.

[18] Servetto SD, Ramchandran K, Orchard MT. Image coding based on a morhpological representation of wavelet data. IEEE Trans Image Proc 1999; 8(9): 1161-74.

[19] Chai B, Vass J, Zhuang X. Significance-linked connected component analysis for wavelet image coding. IEEE Trans Image Proc 1999; 8(6): 774-84.

[20] Joshi RL, Hafarkhani H, Kasner JH, Fisher TR, Farvardin N, Marcellin MW. Comparison of different methods of classification in sub band coding of images. IEEE Trans Image Proc 1997; 6(11): 1473-86.

[21] LoProso SM, Ramchandran K, Orchard MT. Image coding based on mixture modeling of wavelet coefficients and a fast estimationquantization framework. In: Proc. Data Compression Conf. DCC'97 1997; pp. 221-30.

[22] Chrysafis C, Ortega A. Efficient context based entropy coding for lossy wavelet image compression. In: Proc. Data Compression Conf. DCC'97 1997; pp. 241-50.

[23] Jacquin AE. Image coding based on a fractal theory of iterated contractive image transformations. IEEE Trans Image Proc 1992; 1(1): 18-30.

[24] Ning L. Fractal Imaging. US: Academic Press, 1997.

[25] Pentland A, Horowitz B. A practical approach to fractal-based image compression. In: Proc. Data Compression Conf. DCC'91 1991; pp. 176-85.

[26] Rinaldo R, Calvagno G. Image coding by block prediction of multiresolution subimages. IEEE Trans image Proc 1995; 4: 90920 .

[27] Davis G. A wavelet-based analysis of fractal image compression. IEEE Trans Image Proc 1998; 7: 141-54.

[28] Li J, Kuo CC. Image compression with a hybrid wavelet-fractal coder. IEEE Trans Image Proc 1999; 8: 868-74. 
[29] Zukoski M J, Boult T, Iyriboz T. A novel approach to medical image compression. Lect Notes Bioinforma 2006; 2: 89-103.

[30] Chen YT, Tseng DC. Wavelet-based medical image compression with adaptive prediction. Comput Med Imaging Graphic 2007; 31: $1-8$.

[31] Babud V, Alamelu R. Wavelet Based Medical Image Compression Using ROI EZW. Int J Recent Trans Eng 2009; 1: 97-100.

[32] Kumar S, Singh SS. Image compression techniques for medical images: A Review. Int J Res Eng Appl Sci 2012; 1195-9.

[33] Devi PSA, Mini MG. Compression of computed radiographic images using linear prediction on wavelet coefficients. In: ICACC 2012; pp. 130-3.

[34] Singh S, Kumar V, Verma HK. DWT-DCT hybrid scheme for medical image compression. J Med Eng Technol 2007; 31: 109-22.

[35] Al-Fahoum AS, Al-Shamali A. A new adaptive wavelet-based ecg compression technique. In: PACII 2010; vol. 2: pp. 468-71.
[36] Al-Fahoum AS. Quality assessment of ECG compression techniques using a wavelet-based diagnostic measure. IEEE Trans Inf Technol B 2006; 10: 182-92.

[37] Tahoees PG, Varela JR, Lado MJ, Souto M. Image compression: Maxshift ROI encoding options in JPEG2000. Comput Vis Image Underst 2008; 109: 139-45.

[38] Chen YY. Medical image compression using DCT-based subband decomposition and modified SPIHT data organization. Int J Med Inform 2007; 76: 717-25.

[39] Hang X, Greenberg NL, Thomas JD. Compression of pre-scanconverted echocardiographic video using wavelet packet and integer wavelet transforms. Image Vision Comput 2006; 24: 91525.

[40] Al-Fahoum A. Adaptive edge localization approach for QCA. Med Biol Eng Comput 2003; 41(4): 425-32.

(C) Al-Fahoum and Harb; Licensee Bentham Open.

This is an open access article licensed under the terms of the Creative Commons Attribution Non-Commercial License (http://creativecommons.org/licenses/by$\mathrm{nc} / 3.0 /$ ), which permits unrestricted, non-commercial use, distribution and reproduction in any medium, provided the work is properly cited. 